\title{
Extended Sentinel Node Biopsy in Breast Cancer Patients who Achieve Complete Nodal Response with Neoadjuvant Chemotherapy
}

\author{
Alfredo Carlos Simóes Dornellas de Barros (D), Danúbia Ariana de Andrade (iD \\ Hospital Beneficência Portuguesa de São Paulo, Mastology, São Paulo, Brazil
}

\begin{abstract}
Neoadjuvant chemotherapy (NAC) can eradicate axillary disease in breast cancer (BC) patients. Sentinel node biopsy (SNB) in patients with positive axilla who accomplish complete clinical response after NAC is a new opportunity for changing paradigms and decreasing the extension and the morbidity of axillary surgery. The aim of this article is to review the limits of SNB in this setting and present the current status of an expanded modification of this technique. False-negative rates (FNRs) of conventional SNB exceed the threshold of $10 \%$, and are not acceptable. The extended SNB (ESNB) entails the removal of at least 3 lymph nodes (LNs) including the sentinel node (SN) mapped by dual tracers and a marked lymph node (LN) that was found previously metastatic. This node by node removal procedure greatly reduces the FNRs of the procedure. Despite that axillary lymph node dissection (ALND) is still the standard of care for patients with involved LNs before NAC, the ESNB is a valid option for selected patients in whom axillary positive disease is converted to negative. When ESNB is negative in such cases (immunohistochemistry included), the omission of ALND seems to be safe.

Keywords: Breast cancer, neoadjuvant chemotherapy, sentinel node biopsy
\end{abstract}

Cite this articles as: Barros ACSD, Andrade DA. Extended Sentinel Node Biopsy in Breast Cancer Patients who Achieve Complete Nodal Response with Neoadjuvant Chemotherapy. Eur J Breast Health 2020; 16(2): 99-105.

\section{Introduction}

Neoadjuvant chemotherapy (NAC) for breast carcinoma (BC), in combination with personalized targeted therapy, allows for high rates of pathologic complete response (pCR) (1-3).

Sentinel node biopsy (SNB) is safe after successful NAC in patients with clinically-negative axilla (3-5). For BC patients with positive lymph nodes (LNs) prior to NAC the standard surgery for treating the axilla has been axillary lymph node dissection (ALND), but as NAC enables downstaging of axillary disease, SNB in patients with clinically nodes positive $(\mathrm{cN}+)$ axilla who accomplish complete clinical response (yc N0) is an opportunity for changing paradigms.

This article reviews the role of SNB after NAC for BC treatment and presents the current status of a modification of this technique, the extended sentinel node biopsy (ESNB), in patients with initially positive axilla who achieve favorable response with NAC.

Impact of neoadjuvant chemotherapy on nodal burden

Numerous studies have demonstrated the disappearance (on average 40\%) of LNs infiltration after anthracyclines and taxane-based regimens, in association with single or dual anti-HER-2 therapy for patients with HER-2 positive (HER-2+) disease (6-11).

$\mathrm{BC}$ is a heterogeneous disease and the chance of nodal pathologic complete response (pCR) strongly depends on molecular parameters. For Al-Hilli et al. (12), and Mamtani et al. (13), nodal pCR varied greatly based on immunohistochemical (IMH) classification, as shown in Table 1 . The overall rates of nodal pCR were, respectively, $37.7 \%$ and $49.2 \%$ according to these authors. With advancements in the understanding of tumor subtypes, NAC is increasingly focused on HER-2 + and triple negative subtypes and less frequently used in hormone receptor-positive disease.

In the preoperative phase, ensuring that NAC is effective to downstage the LNs, is based on the comparison between nodal evaluation at diagnosis and after NAC, by physical examination and ultrasonography, that is considered the imaging technique of choice to monitor the 
Table 1. Different breast cancer subtypes and nodal pathologic complete response with neoadjuvant chemotherapy

\begin{tabular}{|lcccc} 
& ER+, HER-2 - (\%) & ER+, HER-2 + (\%) & ER-, HER-2 + (\%) & ER-, HER-2 - (\%) \\
\hline Al-Hilli et al. (12) & 20.2 & 47.7 & 61.3 & 47.3 \\
Mamtani et al. (13) & 20.5 & 70.2 & 96.6 & 47.2
\end{tabular}

Table 2. Meta-analyses estimating the false-negative rate (FNR) of the sentinel node (SN) biopsy after neoadjuvant chemotherapy in node-positive patients converted to node-negative

\begin{tabular}{|c|c|c|c|}
\hline & $\mathbf{n}$ & Nodal positivity at diagnosis & FNR (\%) \\
\hline Fu et al. (19) & 2471 & clinical/ultrasonographic & $14 *(95 \% \mathrm{Cl}: 10 \%-17 \%)$ \\
\hline Van Nijnatten et al. (22) & 1395 & microscopic & $15.1^{* *}(95 \%$ Cl: $12.7 \%-17.6 \%)$ \\
\hline El Hage Chehade et al. (23) & 3398 & microscopic & 13 (95\% Cl: 10.8\%-15.6\%) \\
\hline Simons et al. (24) & 2002 & microscopic & $17\left(I^{2}=38.66 \%, p=0.05\right)$ \\
\hline
\end{tabular}

Table 3. False-negative rates of sentinel node biopsy after neoadjuvant chemotherapy in cN+ axilla converted to $\mathrm{cN}$ - axilla

\begin{tabular}{|c|c|c|c|c|c|c|}
\hline & \multirow[b]{2}{*}{$\mathbf{n}$} & \multirow[b]{2}{*}{ Patients } & \multirow[b]{2}{*}{ Overall } & \multicolumn{2}{|c|}{ False-negative rate (\%) } & \multirow[b]{2}{*}{3 SNs } \\
\hline & & & & Dual tracers & $2 \mathrm{SNs}$ & \\
\hline AcosoG Z1071 ${ }^{26}$ & 756 & T0-4, N1-2 & 12.6 & 10.8 & 21.2 & 9.1 \\
\hline SENTINA 25 & 592 (Arm C) & N1-2 & 14.2 & 8.6 & 18.5 & 7.3 \\
\hline SN FNAC 27 & 153 & T0-3, N1-2 & 9.6 & 5.2 & - & $4.9 *$ \\
\hline
\end{tabular}

involution of LNs. This procedure is simple and also helps to guide the fine-needle aspiration or core biopsy of LNs suspected of involvement prior to beginning systemic therapy. In the same ultrasound study, once the node is punctured, a marker may be placed in the lymph node (LN) to help the surgeon find it during surgery (7-16). No imaging technique, however, is precise enough accurate to predict nodal pCR in patients treated with NAC (14-18).

Usual sentinel node biopsy after neoadjuvant chemotherapy in clinically positive axilla at diagnosis

The identification rate of sentinel node (SN) after NAC is quite acceptable $( \pm 90 \%)$, but it is lower than the one observed in upfront surgeries, probably because of fibrotic compression over the lymphatic capillaries (19). The main limitation of SNB in this setting is its high

\section{Key Points}

- $\quad$ Extended sentinel node biopsy is considered oncologically safe for patients with complete clinical response after neoadjuvant chemotherapy.

- The technique involves dual mapping sentinel node, resection of at least 3 lymph nodes, including the metastatic one marked at diagnosis.

- The omission of axillary lymph node dissection is valid option in patients with microscopically negative disease. false negative result (FNR), estimated to be greater than 25\% in initial studies $(20,21)$. Four meta-analyses calculated the FNR in node-positive patients converted to node-negative varying from $13 \%$ to $17 \%$ (Table 2) (19-24). In all studies, the FNR was deemed unacceptable since it was higher than $10 \%$, the threshold adopted, based on the reasoning that is should not be greater than the rate observed in patients without NAC.

Three pivotal observational studies assessed SNB in patients with $\mathrm{cN}+$ axilla who converted to ycN0 status after NAC (Table 3).

The SENTINA (SENTInel NeoAdjuvant study) was undertaken in Germany and Austria (25). In one of the study arms, 592 patients that converted from $\mathrm{cN}+$ to $\mathrm{ycN} 0$ were treated with both $\mathrm{SNB}$ and axillary lymph node dissection (ALND). The SN detection rate was $80.1 \%$, and its FNR was $14.2 \%$. The FNR was $24.3 \%$ for women who had one SN removed, $18.5 \%$ for those who had two sentinel nodes (SNs) removed, and $4.9 \%$ for those who had at least three $\mathrm{SNs}$ removed. The FNR was $8.6 \%$ for patients who underwent dual SN mapping (vital dye and radiocolloid) compared with $16.0 \%$ for those who received radiocolloid alone.

The American study ACOSOG Z1071 determined the FNR of SNB after NAC, in women initially presenting with pathologically confirmed node-positive disease, when at least 2 SNs were excised (26). 
Positive $\mathrm{SN} s$ were defined as those with metastasis larger than $0.2 \mathrm{~mm}$. A total of 701 women $(663 \mathrm{cN} 1$ and $38 \mathrm{cN} 2)$ were enrolled. After completion of NAC, $582(83.0 \%)$ patients lacked palpable LNs. The SNB FNR was $12.6 \%$, exceeding the acceptable threshold of $10 \%$, which did not support the use of SNB in this population. Nevertheless, the authors found the following relevant factors lowering the FNR: a) the FNR was $91 \%$ when $\geq 3 \mathrm{SN}$ were examined; b) the FNR was $20.3 \%$ with single agent mapping and $10.8 \%$ when vital dye and radiocolloid were used; and c) the FNR was $11.3 \%$ when the axilla became clinically negative and $19.2 \%$ when palpable axillary LNs persisted.

In the French SN FNAC study (Sentinel Node Biopsy Following NeoAdjuvant Chemotherapy), 145 patients with biopsy-proven nodepositive disease (T0-3, N1-2) underwent SNB and ALND (27). Pathological examinations using IMH were mandatory if $\mathrm{SN}$ was negative by hematoxylin and eosin (H\&E) staining. The $\mathrm{SN}$ identification rate was $87.6 \%(127 / 145)$, and the axillary pCR was $34.5 \%$. The FNR of SNB was $8.4 \%$.

The usual SNB is not sufficiently accurate in this setting. The highest FNR for SNB in relation to the axillary LNs accepted is $10 \%$, taking into account its estimated value for T1-2 N0 BC cases (28). The prognostic influence of eventual malignant cell permanence in the axillary LNs is a cause of serious concern; experimentally, it was proved in mice, that LNs are foci for systemic dissemination through fine capillaries inside them $(29,30)$.

Kang et al. reviewed the records of 1247 patients who had clinically axillary LN-positive status and presented negative conversion following NAC (31). Patients who underwent axillary surgery with SNBguided decisions were compared with patients who underwent upfront ALND. Kaplan-Meier analysis showed that recurrence-free survival (axilla and distant metastases) were not significantly different between the groups (4-year recurrence-free survival: $97.8 \%$ vs. $99.0 \%, \mathrm{p}=0.14$ ).

Galimberti et al. (32), evaluated 147 women with clinical nodal involvement (cT1-4 and $\mathrm{cN} 1-2$ ), who converted to $\mathrm{cN} 0$, and were submitted to SNB. ALND was not performed if the SN was microscopically unaffected. The SN was negative in $70(47.6 \%)$ patients and positive in $77(52.4 \%)$ patients, that underwent ALND. After a median follow-up of 61 months. Kaplan-Meier curves were not statistically different for distant disease free survival $(\mathrm{p}=0.54)$ and overall survival $(\mathrm{p}=0.72)$ in both groups of patients.

\section{Extended sentinel node biopsy after successful neoadjuvant chemotherapy}

The first practical point for decreasing the FNR of SNB is collecting at least $3 \mathrm{SNs}$, provided the number of LNs obtained is inversely proportional to the FNR after NAC. Several authors demonstrated that it was possible to accomplish a $10 \% \mathrm{FNR}$, when $\geq 3$ negative SNs were withdrawn (25-27). Technique matters, and the detection rate and chance to obtain at least $3 \mathrm{SN}$ s are higher when dual tracer mapping was used. In contrast, random sampling of the axillary nodes is not known to affect the FNR and should not be indicated (33).

Marking nodes containing metastases before NAC and harvesting them along the dual mapped SNs (hot/blue) established ESNB as a new and more accurate method for axillary approach. The procedure entails the combination of dual SN mapping methods with the excision of the marked LN found previously involved and eventual removal of suspicious enlarged nodes. The $\mathrm{SNs}$ are identified by vital dye and radiocolloid and the metastatic LN prior to NAC could be or not one of these structures; anyhow, at least 3 LNs should be retrieved.

\section{Patient selection}

Breast pCR is highly correlated with nodal status after NAC. Tadros et al. (34), in HER-2+ or triple-negative BC patients without a breast pCR, described a relative risk for positive nodal metastases after NAC of 7.4 (95\% CI: 3.7-14.8), compared with those with a breast pCR. Among 237 patients (T1-2) with initial biopsy-proved N1 disease, 77 (32.4\%) presented breast pCR and $160(67.5 \%)$ had residual tumor in the breast whereas only $10.4 \%$ of the patients with breast pCR were found to have residual disease in the axilla. Conversely, in $57.5 \%$ of patients without breast pCR, metastatic LNs were detected. Thus, patients without breast pCR do not seem to be appropriate for omission of ALND after NAC.

The GANEA 2 study assessed the SNB in the neoadjuvant scenario. Among patients with a cytologically proven axillary involvement before NAC the FNR of the SNB was $11.9 \%$ (dual mapping) (35). They found that the amount of residual breast tumor allows identifying patients with a low risk of ALND involvement. For patients with negative SNB and a remaining breast tumor size less than $5.0 \mathrm{~mm}$ and no lymphovascular invasion, the risk of a positive complementary ALND was $3.7 \%$.

With regard to molecular subtypes, apart from the low rates of nodal involution with NAC in patients with luminal tumors, positive estrogen receptor (ER+) and HER-2 negative (HER-2-) the FNR of $\mathrm{SNB}$ in these patients is generally unacceptable. Enokido et al. (36) described the following SNB FNRs in women initially presenting with cytology-proven node positivity for each BC subtype: $42.1 \%$ for ER+, HER-2 - ; 16.7\% for ER+, HER-2 +; 3.2\% for negative estrogen receptor (ER-), HER-2 +; and 10.5\% for ER - , HER-2 -.

Despite the paucity of data about the criteria for eligibility of $\mathrm{cN}_{+}$ patients to ESNB post-NAC, adequate patient selection is paramount for a safe procedure. In our opinion candidates for ESNB after NAC need to fulfill the following conditions:

At diagnosis: non-luminal BC subtypes (ER+HER2+; ER-HER2+; or ER-HER2 -), T1-2, cN1-2a;

After NAC: tumor complete clinical response, and conversion to a cN0 status.

\section{Techniques}

Ensuring the exact removal of the initially involved LN by malignant cells is a challenge for surgeons, because generally there is reduction in size of the downstaged LN. The utmost concerns for a valid ESNB are correct marking of the proven-positive $\mathrm{LN}$ and its precise excision. Thus, normally, the affected LN is tagged under ultrasound guidance, just after the nodal puncture, by inserting a metallic clip and/or a radioactive seed, and/or by injecting a charcoal suspension for tattooing.

In a landmark paper, the authors of the ACOSOG Z1071 study analyzed a subgroup of 170 patients, in whom a clip was placed in the positive LN, and the number of excised LNs was $\geq 2$ (37). The clipped LN was removed separately at surgery, and radiographed to confirm that the LN contained the clip. In 107 (75.9\%) cases, the marked LN was one of the SNs, and in these women the SN FNR was 6.1\%. On the other hand, in $34(24.1 \%)$ cases, the clipped LN was found in the ALND specimen, allowing a FNR of $19.0 \%$. When the clip was 


\section{Table 4. Different methods for marking the positive axillary node}

\begin{tabular}{|c|c|c|}
\hline & Marker & Placement timing \\
\hline Boughey et al. (37) & Titanium clip & Рrechemotherapy \\
\hline Donker et al. (39) & $125 \mathrm{I}$ seed & Prechemotherapy \\
\hline Caudle et al. (40) & $\begin{array}{l}\text { Titanium clip } \\
\text { and }^{125} \text { I seed }\end{array}$ & $\begin{array}{l}\text { Prechemotherapy } \\
\text { and preoperation }\end{array}$ \\
\hline Choy et al. (16) & Charcoal & Prechemothera \\
\hline
\end{tabular}

not found the FNR was $14.3 \%$. For comparison, in another subgroup of 355 patients, without clip placement, the FNR was $13.4 \%$. It was concluded that ESNB, including the presence of the clipped LN in the removed tissue, significantly reduces the FNR.

Caudle et al. (7) performed ALND in 191 patients with biopsy-proven axillary nodes in which a clip was inserted. Nodal disease disappeared after NAC in 71 (33\%) patients. In 118 patients undergoing SNB and ALND, the FNR was $10.1 \%$. The clipped node contained metastasis in 115 patients, resulting in a FNR of $4.2 \%$ for the marked LN in relation to ALND. When the evaluation was performed for the combination of SN biopsies and clipped nodes, the FNR was markedly reduced to $1.4 \%$. Clipped nodes were not seen in $23.1 \%$ (31/134 of patients), including six with negative $\mathrm{SN}$ s and metastasis in the clipped node. Accordingly, clipping positive nodes for extended SNB refines the pathologic evaluation and reduces the FNR.

Cabioglu et al. (38), in a prospective registry trial combining conventional SNB with clipped node excision, ascertained that the clipped node was the $\mathrm{SN}$ in $81.4 \%$ of the cases, whereas in $18.6 \%$ the clipped node was a non-SN. The possibility of clip migration was evidenced, and in $3.5 \%$ the clipped node could not be found in the specimen.

The intraoperative localization of the clipped LN is not an easy task. Therefore, other methodologies to mark the LN have been investigated, such as the placement of a Iodine-125 (125I) seed in the biopsyproven positive $\mathrm{LN}$. The node with the seed is posteriorly excised using a hand held gamma detection probe during the surgery. Donker et al. (39) described an identification rate of the seed-containing LN of $97 \%$ with this technique.

Caudle et al. (40), from the MD Anderson Cancer Center, proposed a variation of this procedure, the Targeted Axillary Dissection (TAD) method. This methodology consists of SNB and excision of the positive LN, which was first marked by a clip prior to chemotherapy, and months later, before surgery, was additionally tagged by a ${ }^{125} \mathrm{I}$ seed. A gamma probe optimized to ${ }^{125} \mathrm{I}$ activity, which is distinct from the setting used in the SN identification with Technetium-99m (99mTc), guides the removal of the LN marked with the clip and seed. Intraoperative radiography is performed to check for the presence of both markers in the tissue sample before pathologic handling. They had a FNR of $2 \%$ with TAD versus $10.6 \%$ with SNB alone.

Diego et al. (41) used the same methodology in 30 patients, and they described intraoperative finding of blue dye or ${ }^{99 \mathrm{~m}}$ Tc activity in the ${ }^{125}$ I-localized LN in $73.3 \%$ of the cases.

Another strategy, proposed by Choy et al. (16), is to inject a small cortex of the LN and adjacent soft tissue just after the nodal fine needle aspiration. The tattooed node is visible intraoperatively even months later. Park et al. (42) approved the diagnostic performance of SNB using the technique of charcoal tattooing of cytologic-metastatic LN at presentation. The carbon nodal injection may occur before NAC, exclusive or combined with the clip, or later, preoperatively, in nodes previously marked by a clip.

The concordance between the pathologic results for both the charcoal tattooed nodes and the nodes containing blue dye or radiocolloid was analyzed by Kim et al. (43). In 45 cases the tattooed nodes were not identified in the surgical field in only 1 (2.2\%). In 25 cases $(56.8 \%)$ there was concordance between the $\mathrm{SN}$ and the tattooed node, they were the same structure. In the final pathological results, 18 (40.0\%) patients had metastatic nodes. The sensitivities of the SNB, charcoal marked node biopsy, and the combination radiocolloid and/or tattooed node biopsy, for axillary metastasis identification, were $61.1 \%$, $66.6 \%$, and $77.8 \%$, respectively.

The different techniques, employed in order to facilitate the identification of the node previously containing malignant cells in the surgical field, are summarized on Table 4. All of them entail the placement of a marker under ultrasound guidance. Each method has pros and cons.

A clipped lymph node is not easily identified during the surgery, since it is not visible nor detected by a probe. A nodal radiography is mandatory to confirm the harvesting precision. Besides, we must consider that hazardous spontaneous clip migration is possible. On its turn the radioisotopic seed placing is more complex and requires interaction between the services of Nuclear Medicine and Ultrasonography. Moreover, the 5-6 months permanence of a radioactive source in the human body is theoretically undesirable, and in some countries federal laws do not allow this type of procedure. To circumvent this problem, in the TAD method the ${ }^{125} \mathrm{I}$ seed is placed in a second-step procedure after NAC, some days prior the surgery.

The method of node tattooing with black carbon is simple, cheap, and the ink injection could be done at the same moment of the puncture for cytology/histopathology. It is easier to find a tattooed node than a clipped one, and the excised node radiography is not needed. The sole precaution is not to inject excessive volume of carbon suspension inside the node, since it may interfere with the microscopic analysis.

The value of the isolated tumor cells and micrometastases in the sentinel node

Low-volume SN disease after NAC is not an indicator of a low risk of additional positive axillary nodes. In contrast with its role in adjuvant treatment, it is likely that isolated tumor cells (ITCs) or micrometastasis in the SN after NAC, remnants of nodal disease that were not sensitive to chemotherapy, have a different meaning, and could have a negative impact on the evolution of the patients. Of note, patients with micrometastases in the $\mathrm{SN}$ after NAC have high rates (12\%-64\%) of positive non-SNs (44-46).

In the SN FNAC trial, FNR improved from $13.3 \%$ to $8.4 \%$ when IMH had been used (27). The importance of IHC was also evident in the ACOSOG Z1071 study: the trial's FNR was $8.7 \%$ when any ITCpositive node was included compared with $12.6 \%$ without IHC (37).

For Moo et al. (46) $17 \%$ of the patients with ITCs and $64 \%$ of the patients with micrometastasis in the $\mathrm{SN}$ had additional nodal metastases at ALND. 
A meta-analysis conducted by Fu et al. (19) estimated that the SN FNR reduced from $16.0 \%$ to $8.7 \%$, if ITC detected by the additional IHC, was considered positive.

There are typical findings in the metastatic LNs which had negative conversion after NAC. Chemotherapy effects on the positive node include fibrosis, hemosiderin deposits, laden macrophages, increased vascularity and a foamy histiocytic infiltrate. Malignant cells may be scattered through a fibrotic LN, and cytokeratins-IMH may be useful to confirm suspicious morphology (47). In clinical practice, cytokeratin staining should be added to achieve a more accurate ESNB result when the excised $\mathrm{LN}$ s are negative according to $\mathrm{H} \& \mathrm{E}$ analysis $(9,48)$.

Intraoperative evaluation of SNs could prevent a second surgery by indicating immediate ALND. Rubio et al. (45), after performing SNB and completing ALND in the same patients after NAC, demonstrated that frozen sectioning of the SNs is effective, with sensitivity ranging from $78.5 \%$ for micrometastasis and ITC to $100 \%$ for macrometastasis. In the experience of the Memorial Sloan Kettering Cancer Center the FNR of the frozen section of the SN was $6.2 \%$ and minimal involvement of the $\mathrm{SN}$ in the final pathology was an indication for ALND (46).

\section{Conclusion}

ALND is still the standard procedure for the management of the axilla of BC patients with metastatic lymph nodes before NAC. Nevertheless, ESNB (dual SN mapping, resection of at least $3 \mathrm{LNs}$, including the metastatic one marked at diagnosis) is considered a valid option for selected patients with axillary positive disease that is converted to negative after NAC. In patients with negative microscopic findings in the excised LNs (IMH included), the omission of ALND seems to be oncologically safe. Future research studies focusing specifically on prognosis of the treated patients are needed for definitive judgement.

Peer-review: Externally peer-reviewed.

Author Contributions: Concept - A.C., D.A.; Design - A.C., D.A.; Supervision - A.C., D.A.; Resources - A.C., D.A.; Materials - A.C., D.A.; Data Collection and/or Processing - A.C., D.A.; Analysis and/or Interpretation - A.C., D.A.; Literature Search - A.C., D.A.; Writing Manuscript - A.C., D.A.; Critical Review - A.C., D.A.

Conflict of Interest: The authors have no conflicts of interest to declare.

Financial Disclosure: The authors declared that this study has received no financial support.

\section{References}

1. Rastogi P, Anderson SJ, Bear HD, Geyer CE, Kahlenberg MS, Robidoux A, et al. Preoperative chemotherapy: updates of National Surgical Adjuvant Breast and Bowel Project Protocols B-18 and B-27. J Clin Oncol 2008; 26: 778-785. (PMID: 18258986) [CrossRef]

2. King TA, Morrow M. Surgical issues in patients with breast cancer receiving neoadjuvant chemotherapy. Nat Rev Clin Oncol 2015; 12: 335-343. (PMID: 25850554) [CrossRef]

3. Mamounas EP, Brown A, Anderson S, Smith R, Julian T, Miller B, et al. Sentinel node biopsy after neoadjuvant chemotherapy in breast cancer: results from National Surgical Adjuvant Breast and Bowel Project Protocol B-27. J Clin Oncol 2005; 23: 2694-2702. (PMID: 15837984) [CrossRef]
4. Hunt KK, Yi M, Mittendorf EA, Guerrero C, Babiera GV, Bedrosian I, et al. Sentinel lymph node surgery after neoadjuvant chemotherapy is accurate and reduces the need for axillary dissection in breast cancer patients. Ann Surg 2009; 250: 558-566. (PMID: 19730235)

5. Geng C, Chen X, Pan X, Li J. The feasibility and accuracy of sentinel lymph node biopsy in initially clinically node-negative breast cancer after neoadjuvant chemotherapy: a systematic review and meta-analysis. PLoS One 2016; 11: e0162605. (PMID: 27606623) [CrossRef]

6. Fan Z, Li J, Wang T, Xie Y, Fan T, Lin B, et al. Level III axillary lymph nodes involvement in node positive breast cancer received neoadjuvant chemotherapy. Breast 2013; 22: 1161-1165. (PMID: 24080493) [CrossRef]

7. Caudle AS, Yang WT, Krishnamurthy S, Mittendorf EA, Black DM, Gilcrease MZ, et al. Improved axillary evaluation following neoadjuvant therapy for patients with node-positive breast cancer using selective evaluation of clipped nodes: implementation of targeted axillary dissection. J Clin Oncol 2016; 34: 1072-1078. (PMID: 26811528) [CrossRef]

8. Yu Y, Cui N, Li HY, Wu YM, Xu L, Fang M, et al. Sentinel lymph node biopsy after neoadjuvant chemotherapy for breast cancer: retrospective comparative evaluation of clinically axillary lymph node positive and negative patients, including those with axillary lymph node metastases confirmed by fine needle aspiration. BMC Cancer 2016; 16: 808. (PMID: 27756234) [CrossRef]

9. Park S, Lee JE, Paik HJ, Ryu JM, Bae SY, Lee SK, et al. Feasibility and prognostic effect of sentinel lymph node biopsy after neoadjuvant chemotherapy in cytology-proven, node-positive breast cancer. Clin Breast Cancer 2017; 17: e19-e29. (PMID: 27495997) [CrossRef]

10. Mougalian SS, Hernandez M, Lei X, Lynch S, Kuerer HM, Symmans WF, et al. Ten-year outcomes of patients with breast cancer with cytologically confirmed axillary lymph node metastases and pathologic complete response after primary systemic chemotherapy. JAMA Oncol 2016; 2: 508-516. (PMID: 26720612) [CrossRef]

11. Schneeweiss A, Chia S, Hickish T, Harvey V, Eniu A, Hegg R, et al. Pertuzumab plus trastuzumab in combination with standard neoadjuvant anthracycline-containing and anthracycline-free chemotherapy regimens in patients with HER-2 positive early breast cancer: a randomized phase II cardiac safety study (THYPHAENA). Ann Oncol 2013; 24: 2278-2284. (PMID: 23704196) [CrossRef]

12. Al-Hilli Z, Hoskin TL, Day CN, Habermann EB, Boughey JC. Impact of neoadjuvant chemotherapy on nodal disease and nodal surgery by tumor subtype. Ann Surg Oncol 2018; 25: 482-493. (PMID: 29181679) [CrossRef]

13. Mamtani A, Barrio AV, King TA, Van Zee KJ, Plitas G, Pilewskie, et al. How often does neoadjuvant chemotherapy avoid axillary dissection in patients with histologically confirmed nodal metastases? Results of a prospective study. Ann Surg Oncol 2016; 23: 3467-3474. (PMID: 27160528) [CrossRef]

14. Ruano Pérez R, Rebollo Aguirre AC, García-Talavera San Miguel P, Díaz Expósito R, Vidal-Sicart S, Cordero García JM, et al. Review of the role of the sentinel node biopsy in neoadjuvant chemotherapy in women with breast cancer and negative or positive axillary node at diagnosis. Rev Esp Med Nucl Imagen Mol 2018; 37: 63-70. (PMID: 28869178) [CrossRef]

15. Shin K, Caudle AS, Kuerer HM, Santiago L, Candelaria RP, Dogan B, et al. Radiologic mapping for targeted axillary dissection: needle biopsy to excision. Am J Roentgenol 2016; 207: 1372-1379. (PMID: 27726422) [CrossRef]

16. Choy N, Lipson J, Porter C, Ozawa M, Kierin A, Pal S, et al. Initial results with preoperative tattooing of biopsied axillary lymph nodes and correlation to sentinel lymph nodes in breast cancer patients. Ann Surg Oncol 2015; 22: 377-382. (PMID: 25164040) [CrossRef]

17. Diepstraten SC, Sever AR, Buckens CF, Veldhuis WB, van Dalen T, van den Bosch MA, et al. Value of preoperative ultrasound-guided axillary lymph node biopsy for preventing completion axillary lymph node dissection in breast cancer: a systematic review and meta-analysis. Ann Surg Oncol 2014; 21: 51-59. (PMID: 24008555) [CrossRef] 
18. Cooper KL, Meng Y, Harnan S, Ward SE, Fitzgerald P, Papaioannou D, et al. Positron emission tomography (PET) and magnetic resonance imaging (MRI) for the assessment of axillary lymph node metastases in early breast cancer: systematic review and economic evaluation. Health Technol Assess 2011; 15: 1-134. (PMID: 21276372) [CrossRef]

19. Fu JF, Chen HL, Yang J, Yi CH, Zheng S. Feasibility and accuracy of sentinel lymph node biopsy in clinically node-positive breast cancer after neoadjuvant chemotherapy: a meta-analysis. PLoS One 2014; 9: e105316. (PMID: 25210779) [CrossRef]

20. Shen J, Gilcrease MZ, Babiera GV, Ross MI, Meric-Bernstam F, Feig BW, et al. Feasibility and accuracy of sentinel lymph node biopsy after preoperative chemotherapy in breast cancer patients with documented axillary metastases. Cancer 2007; 109: 1255-1263. (PMID: 17330229) [CrossRef]

21. Alvarado R, Yi M, Le-Petross H, Gilcrease M, Mittendorf EA, Bedrosian I, et al. The role for sentinel lymph node dissection after neoadjuvant chemotherapy in patients who present with node-positive breast cancer. Ann Surg Oncol 2012; 19: 3177-3184. (PMID: 22772869) [CrossRef]

22. van Nijnatten TJ, Schipper RJ, Lobbes MB, Nelemans PJ, Beets-Tan RG, Smidt ML. The diagnostic performance of sentinel lymph node biopsy in pathologically confirmed node positive breast cancer patients after neoadjuvant systemic therapy: a systematic review and meta-analysis. Eur J Surg Oncol 2015; 41: 1278-1287. (PMID: 26329781) [CrossRef]

23. El Hage Chehade H, Headon H, El Tokhy O, Heeney J, Kasem A, Mokbel K. Is sentinel lymph node biopsy a viable alternative to complete axillary dissection following neoadjuvant chemotherapy in women with node-positive breast cancer at diagnosis? An updated meta-analysis involving 3, 398 patients. Am J Surg 2016; 212: 969-981. (PMID: 27671032) [CrossRef]

24. Simons JM, van Nijnatten TJA, van der Pol CC, Luiten EJT, Koppert LB, Smidt ML. Diagnostic accuracy of different surgical procedures for axillary staging after neoadjuvant systemic therapy in node-positive breast cancer: a systematic review and meta-analysis. Ann Surg 2019; 269: 432442. (PMID: 30312200) [CrossRef]

25. Kuehn T, Bauerfeind I, Fehm T, Fleige B, Hausschild M, Helms G, et al. Sentinel-lymph-node biopsy in patients with breast cancer before and after neoadjuvant chemotherapy (SENTINA): a prospective, multicentre cohort study. Lancet Oncol 2013; 14: 609-618. (PMID: 23683750) [CrossRef]

26. Boughey JC, Suman VJ, Mittendorf EA, Ahrendt GM, Wilke LG, Taback B, et al. Sentinel lymph node surgery after neoadjuvant chemotherapy in patients with node-positive breast cancer: the ACOSOG Z1071 (Alliance) clinical trial. JAMA 2013; 310: 1455-1461. (PMID: 24101169) [CrossRef]

27. Boileau JF, Poirier B, Basik M, Holloway CM, Gaboury L, Sideris L, et al. Sentinel node biopsy after neoadjuvant chemotherapy in biopsy-proven node-positive breast cancer: the SN FNAC study. J Clin Oncol 2015; 33: 258-264. (PMID: 25452445) [CrossRef]

28. Krag DN, Anderson SJ, Julian TB, Brown AM, Harlow SP, Ashikaga T, et al. Technical outcomes of sentinel-lymph node resection and conventional axillary-lymph-node dissection in patients with clinically node-negative breast cancer: results from the NSABP B-32 randomised phase III trial. Lancet Oncol 2017; 8: 881-888. (PMID: 17851130) [CrossRef]

29. Pereira ER, Kedrin D, Seano G, Gautier O, Meijer EFJ, Jones D, et al. Lymph node metastases can invade local blood vessels, exit the node, and colonize distant organs in mice. Science 2018; 359: 1403-1407. (PMID: 29567713) [CrossRef]

30. Brown M, Assen FP, Leithner A, Abe J, Schachner H, Asfour G, et al. Lymph node bloode vessels provide exit routes for metastatic tumor cell dissemination in mice. Science 2018; 359: 1408-1411. (PMID 29567714) [CrossRef]

31. Kang YJ, Han W, Park S, You JY, Yi HW, Park S, et al. Outcome following sentinel lymph node biopsy-guided decisions in breast cancer patients with conversion from positive to negative axillary lymph nodes after neoadjuvant chemotherapy. Breast Cancer Res Treat 2017; 166: 473-480. (PMID: 28766131) [CrossRef]
32. Galimberti V, Ribeiro Fontana SK, Maisonneuve P, Steccanella F, Vento $A R$, Intra $M$, et al. Sentinel node biopsy after neoadjuvant treatment in breast cancer: five-year follow-up of patients with clinically node-negative or node-positive disease before treatment. Eur J Surg Oncol 2016; 42: 361-368. (PMID: 26746091) [CrossRef]

33. Rubio IT. Sentinel lymph node biopsy after neoadjuvant treatment in breast cancer: work in progress. Eur J Surg Oncol 2016; 42: 326-332. (PMID: 26774943) [CrossRef]

34. Tadros AB, Yang WT, Krishnamurthy S, Rauch GM, Smith BD, Valero $\mathrm{V}$, et al. Identification of patients with documented pathologic complete response in the breast after neoadjuvant chemotherapy for omission of axillary surgery. JAMA Surg 2017; 152: 665-670. (PMID: 28423171) [CrossRef]

35. Classe JM, Loaec C, Gimbergues P, Alran S, de Lara CT, Dupre PF, et al. Sentinel lymph node biopsy without axillary lymphadenectomy after neoadjuvant chemotherapy is accurate and safe for selected patients: the GANEA 2 Study. Breast Cancer Res Treat 201; 173: 343-352. (PMID: 30343457) [CrossRef]

36. Enokido K, Watanabe C, Nakamura S, Ogiya A, Osako R, Akiyama F, et al. Sentinel lymph node biopsy after neoadjuvant chemotherapy in patients with an initial diagnosis of cytology-proven lymph node-positive breast cancer. Clin Breast Cancer 2016; 16: 299-304. (PMID: 26993216) [CrossRef]

37. Boughey JC, Ballman KV, Le-Petross HT, McCall LM, Mittendorf EA, Ahrendt GM, et al. Identification and resection of clipped node decreases the false-negative rate of sentinel lymph node surgery in patients presenting with node-positive breast cancer (T0-T4, N1-N2) who receive neoadjuvant chemotherapy: results from ACOSOG Z1071 (Alliance). Ann Surg 2016; 263: 802-807. (PMID: 26649589) [CrossRef]

38. Cabığlu N, Karanlık H, Kangal D, Özkurt E, Öner G, Sezen F, et al. Improved false-negative rates with intraoperative identification of clipped nodes in patients undergoing sentinel lymph node biopsy after neoadjuvant chemotherapy. Ann Surg Oncol 2018; 25: 3030-3036. (PMID: 29978371) [CrossRef]

39. Donker M, Straver ME, Wesseling J, Loo CE, Schot M, Drukker CA, et al. Marking axillary lymph nodes with radioactive iodine seeds for axillary staging after neoadjuvant systemic treatment in breast cancer patients: the MARI procedure. Ann Surg 2015; 261: 378-382. (PMID: 24743607) [CrossRef]

40. Caudle AS, Yang WT, Mittendorf EA, Black DM, Hwang R, Hobbs B, et al. Selective surgical localization of axillary lymph nodes containing metastases in patients with breast cancer: a prospective feasibility trial. Jama Surg 2015; 150: 137-143. (PMID: 25517573) [CrossRef]

41. Diego EJ, McAuliffe PF, Soran A, McGuire KP, Johnson RR, Bonaventura $\mathrm{M}$, et al. Axillary staging after neoadjuvant chemotherapy for breast cancer: a pilot study combining sentinel lymph node biopsy with radioactive seed localization of pre-treatment positive axillary lymph nodes. Ann Surg Oncol 2016; 23: 1549-1553. (PMID: 26727919). [CrossRef]

42. Park S, Koo JS, Kim GM, Sohn J, Kim SI, Cho YU, et al. Feasibility of charcoal tattooing of cytologic-proven metastatic axillary lymph node at diagnosis and sentinel lymph node biopsy after neoadjuvant chemotherapy in breast cancer patients. Cancer Res Treat 2018; 50: 801-812. (PMID: 28814071) [CrossRef]

43. Kim WH, Kim HJ, Jung JH, Park HY, Lee J, Kim WW, et al. Ultrasoundguided restaging and localization of axillary lymph nodes after neoadjuvant chemotherapy for guidance of axillary surgery in breast cancer patients: experience with activated charcoal. Ann Surg Oncol 2018; 25: 494-500. (PMID: 29134374) [CrossRef]

44. van der Heiden-van der Loo M, de Munck L, Sonke GS, van Dalen T, van Diest PJ, van den Bongard HJ, et al. Population based study on sentinel node biopsy before or after neoadjuvant chemotherapy in clinically node negative breast cancer patients: Identification rate and influence on axillary treatment. Eur J Cancer 2015; 51: 915-921. (PMID: 25857549) [CrossRef] 
45. Rubio IT, Aznar F, Lirola J, Peg V, Xercavins J. Intraoperative assessment of sentinel lymph nodes after neoadjuvant chemotherapy in patients with breast cancer. Ann Surg Oncol 2010; 17: 235-239. (PMID: 19777186) [CrossRef]

46. Moo TA, Edelweiss M, Hajiyeya S, Stempel M, Raiss M, Zabor EC, et al. Is low-volume disease in the sentinel node after neoadjuvant chemotherapy as indication for axillary dissection? Ann Surg Oncol 2018; 25: 1488-1494. (PMID: 29572705) [CrossRef]

47. Krishnamurthy S, Meric-Bernstam F, Lucci A, Hwang RF, Kuerer HM, Babiera G, et al. A prospective study comparing touch imprint cytology, frozen section analysis, and rapid cytokeratin immunostain for intraoperative evaluation of axillary sentinel lymph nodes in breast cancer. Cancer 2009; 115: 1555-1562. (PMID: 19195040) [CrossRef]

48. van Nijnatten TJ, Simons JM, Moossdorff M, de Munck L, Lobbes MB, van der Pol CC, et al. Prognosis of residual axillary disease after neoadjuvant chemotherapy in clinically node-positive breast cancer patients: isolated tumor cells and micrometastases carry a better prognosis than macrometastases. Breast Cancer Res Treat 2017; 163: 159-166. (PMID: 28213782). [CrossRef] 